\title{
Fromagerie de demain (1)
}

\author{
par \\ G. LOFTUS HILLS \\ Département de la Recherche Laitière, C.S.I.R.O., Melbourne
}

\section{1) Vers la mécanisation}

J'avais à l'origine prévu, au cours de cet exposé, de m'occuper en priorité de ce que l'on pourrait appeler « l'autre introduction » à la mécanisation de l'industrie fromagère. Dans notre étude détaillée réalisée à la Section de Recherches laitières du C.S.I.R.O. concernant la mécanisation dans la fabrication du Cheddar et d'un certain nombre d'autres variétés de fromages à pâte dure, nous nous en sommes toujours fermement tenus au principe suivant lequel on ne doit rien tolérer qui soit susceptible de modifier les caractéristiques d'un fromage sous prétexte de rendre notre tâche plus aisée.

Les différentes variétés de fromages fabriqués ici ou là ne constituent pas l'un des moindres attraits de cet aliment, d'autant plus que les consommateurs sont toujours plus nombreux et plus exigeants. C'est un privilège remarquable que le fromage et le vin détiennent seuls. On doit en attendre beaucoup du point de vue de la consommation ; aussi est-il important pour les ventes que ces variétés soient effectivement maintenues.

La mécanisation à peu de frais peut fort bien affecter la qualité et beaucoup penseront que tel a dû être le cas de notre industrie boulangère.

Il est tout aussi vrai que certains procédés, chimiquement sûrs, doivent être écartés pour ne pas déroger au principe du maintien des caractéristiques des diverses variétés de fromages.

"L'autre introduction » à laquelle je faisais allusion consisterait à tirer parti de la meilleure technique chimique pour produire un fromage de qualité, qui serait évidemment, puisque élaboré d'une façon nouvelle, différent des autres. L'inconvénient bien sûr résulterait de ce qu'il aurait à affronter, ainsi que tout produit nouveau, les problèmes du marché. Pour lui, en fait, son marché serait à

(1) Exposé à la Division de Queensland de A.I.D.F.M.S., le 24 juin 1970. 
créer. Ce n'est pas insurmontable, et le prix de revient pourrait justifier le temps et les dépenses nécessaires à son lancement.

Il va sans dire que cette entreprise serait vouée à l'échec si le fromage en lui-même n'avait rien qui retînt l'attention, et « l'autre introduction " n'a de valeur que si, pour l'entreprendre, on comprend réellement ce qu'est l'industrie du fromage et si on peut y déployer autant d'ingéniosité qu'on aurait pu le faire dans, par exemple, l'industrie chimique.

Cependant, si l'essentiel de ce que j'ai à dire concernant les développements de l'industrie fromagère en de nouvelles zones, de ses nouvelles applications et de ses nouvelles techniques, est lié à cette nouvelle approche, une partie néanmoins se rapporte parfaitement à la fabrication de variétés existant à l'heure actuelle. Il vous sera aisé, je pense, de mettre de côté les progrès possibles, ceux qui ne concernent que les méthodes de fabrication des variétés actuelles et ceux qui ne concernent que les méthodes envisagées pour l'avenir.

\section{2) Du meilleur lait pour la fromagerie}

Commençons par considérer les moyens dont nous disposons ou dont nous pourrions disposer pour assurer à l'approvisionnement en lait la meilleure aptitude possible à la fromagerie.

Il est curieux de constater que cette aptitude dépend de la race. Les Russes et les Japonais ont étudié cette question ; par contre en Australie et en Nouvelle-Zélande, elle n'a soulevé que peu d'intérêt. D'autres facteurs jouent : par exemple, la capacité plus ou moins grande des races frisonnes, jerseyaises ou A.I.S. à transformer l'herbe en constituants du lait est le souci du fermier, encore qu'il puisse être intéressé par la production de viande un jour ou l'autre. Mais même en ce domaine le fermier ne dispose pas de données expérimentales auxquelles il puisse avoir recours. Et ce qui est certain, c'est que nous ignorons tout des différentes aptitudes de ces laits, sauf en ce qui concerne la teneur brute en matière grasse et en caséine. La taille des micelles de caséine dispersées dans le lait varie suivant les races. Ces races et même des variétés à l'intérieur de ces races diffèrent entre elles par les variantes génétiques des diverses fractions de caséine qui s'y trouvent. Il a été prouvé que la stabilité à la chaleur de la caséine s'en trouve altérée et il serait étonnant que ce fait soit sans conséquence pour l'aptitude du lait à la fabrication du fromage. La proportion des fractions de caséine est aussi fonction de la saison.

Un point a été très nettement éclairci ces dernières années par les travaux des laboratoires Northfield d'Adelaïde ainsi qu'ailleurs : le lait provenant de vaches atteintes de mammite sub-clinique est impropre à la fabrication du fromage. Le caillé a tendance a être très doux. Les chercheurs d'Adélaïde ont montré que ce phénomène était dû à la présence dans le lait de très petites quantités d'albu- 
mine du sérum sanguin chaque fois qu'il y a infection sub-clinique du pis. Des programmes de contrôle de la mammite, en vigueur dans d'autres Pays, se sont avérés très efficaces pour diminuer ces infections sub-cliniques des troupeaux laitiers.

L'aptitude du lait dépend aussi très étroitement de la proportion de gaz dissous, en particulier de dioxyde de carbone, ce qui a récemment été souligné par le Dr Claessons à Uppsala (Suède), dans un article pour le prochain Congrès du Lait. Une plus forte teneur en dioxyde de carbone donne une coagulation beaucoup plus rapide, cette modification étant plus importante que celle entraînée par Ie changement de $\mathrm{pH}$ qui l'accompagne. Nous savons aussi que la présence d'oxygène bloque le développement des ferments lactiques. Aussi l'élimination de l'oxygène et l'apport de dioxyde de carbone semblent être des données importantes pour la préparation du lait destiné à la fromagerie. Certaines usines aux U.S.A. exploitent à cette fin du lait traité sous vide.

Le degré de $\mathrm{pH}$ initial du lait a aussi une grande importance sur la fabrication. La question ici devient plus compliquée. Le degré de $\mathrm{pH}$ influe sur l'activité de la présure, modifie la libération des sels de calcium dont dépend la coagulation du lait, change la solution des éléments inorganiques à l'intérieur du coagulum et, partant, la composition du sérum qui s'écoule du caillé dès que celui-ci est tranché, ainsi que, bien entendu, la composition de ce petit-lait à partir de ce moment. Ce qui revient à dire que la composition du fromage s'en trouve modifiée puisque tout ce qui ne s'écoule pas avec le petit-lait demeure dans le caillé. La proportion de calcium en particulier, mais aussi des autres composants inorganiques, influent grandement sur la fermeté ou la mollesse du fromage ainsi que sur ses autres données physiques. Si on abaisse le degré de $\mathrm{pH}$ en introduisant un levain dans le lait, par affinage anticipé, on n'augmente pas seulement les chances d'infection pour le bactériophage, on détermine aussi à l'avance pour une large part le taux d'acidité ultérieure. La croissance du levain en vue de donner le degré optimum de $\mathrm{pH}$ au départ ne correspond pas nécessairement au degré souhaitable pour la meilleure production d'acide pour le cours de la fabrication. Aussi il paraît préférable de contrôler le degré de $\mathrm{pH}$ idéal par d'autres moyens, par exemple, à l'aide du dioxyde de carbone.

Des travaux récents effectués en Egypte suggèrent qu'une teneur convenable du lait en éléments traceurs est susceptible d'accélérer le processus de maturation, et si cette hypothèse se confirme, la correction des micro-éléments du lait peut devenir aussi une étape normale de sa préparation en vue de la fabrication du fromage.

\section{3) Teneur en germes}

Les exigences microbiologiques du lait destiné aux fromageries se réduisent au plus petit nombre possible d'organismes vivants, et, 
à cette fin, le fabricant ne se contente pas de rechercher un lait pauvre en germes, il le pasteurise. Ce qui ne veut pas dire qu'on a ainsi le meilleur fromage. Je pense comme les vieux habitués que le bon et antique Cheddar fait avec du lait cru avait bien meilleur saveur. Mais pas toujours, et là est la difficulté. Les bactéries et enzymes qui se trouvaient là par hasard contribuaient parfois à rendre le fromage savoureux, mais il arrivait qu'elles aient l'effet inverse, sans compter le danger des germes pathogènes. Aussi, pour assurer au moins une saveur qui ne réserve pas de surprises et aussi pour rendre le fromage propre à la consommation, la propreté du lait et sa pasteurisation sont devenues des nécessités.

Un procédé récent, toutefois, permet d'espérer qu'il sera possible de réconcilier le meilleur de la saveur du lait cru - pour autant que cela soit dû à la présence d'enzymes - avec une hygiène poussée. Tel est le procédé de bactofugation. On soumet le lait à une force centrifuge puissante dans une sorte d'épurateur dont le bol est muni d'ouvertures. Lorsque le lait passe dans le bol environ 1 p. 100 en est rejeté, ce qui entraîne l'élimination de la plupart des germes, environ 95 p. 100 . Ainsi la bactofugation peut s'employer pour obtenir un lait propre sans recourir aux méthodes de chauffage nécessitées par la pasteurisation. Son application à l'industrie fromagère a été empêchée car la perte est élevée, la baisse de rendement du fromage étant supérieure de beaucoup à la perte de 1 p. 100 de lait. Cependant, l'an passé, la Compagnie Alfa-Laval a mis en application un procédé de stérilisation du produit de bactofugation qui est alors reversé dans le lait, de telle sorte que le rendement demeure inchangé. Une installation de ce type a été mise en service en Finlande pour la fabrication d'un fromage de type Emmental, et une autre doit suivre sous peu, dans un but expérimental à l'usine fromagère Camperdown de Victoria.

Des propositions très différentes pour le pré-traitement du lait ont été faites par une étude récente menée à l'Institut GilbertChandler de Technologie laitière de Werribie. On a découvert que des températures de traitement beaucoup plus élevées donnent de meilleurs rendements, comme on pourrait s'y attendre en raison de l'action réciproque bien connue du chauffage sur les protéines du petit-lait, la lactoglobuline $\beta$ et la caséine. On a avancé autrefois qu'une saveur amère résultait d'un tel traitement, mais nous en reparlerons. Le point qu'il faut souligner est le suivant : le caillé provenant d'un tel lait ne se prête pas au traitement présenté pour la fabrication du Cheddar, mais il permet la production d'un fromage de qualité satisfaisante. Il semble qu'une plus forte température dans le pré-traitement du lait constitue une étape souhaitable pour " l'autre introduction " à la mécanisation dans l'industrie fromagère. Mais il est vraisemblable aussi que l'on doive faire appel à des corrections en ce qui concerne la destruction des enzymes et quelques autres modifications physiques et chimiques qui accompagnent le chauffage du lait à très forte température. Le champ d'investiga- 
tions ici est illimité. Que savons-nous des conséquences pour le caillé d'une homogénéisation à basse pression après un tel chauffage ? Que savons-nous des conséquences de correction du degré de $\mathrm{pH}$ avant le pré-chauffage et des possibilités de nouvelles corrections ensuite ?

\section{La coagulation}

Une quantité importante de " présures » microbiennes est apparue ces dernières années. Leur développement est important non seulement dans la mesure où elles se présentent comme un substitut de la présure de veau moins coûteux et plus facile à obtenir, mais aussi dans la mesure où ces complexes diastasiques sont en général doués de propriétés protéolytiques plus importantes que la classique présure. Leur emploi et l'étude de leur action sur la dégradation des protéines du fromage est susceptible d'entraîner un contrôle plus efficace de ce facteur important de la maturation. L'emploi de certaines de ces présures a eu comme conséquence, du moins dans une faible proportion, d'augmenter l'amertume du fromage. Nous en reparlerons plus loin.

Un point intéressant serait de savoir si l'on doit considérer l'emploi de la coagulation continue comme un trait essentiel de la fabrication du fromage dans les années à venir. Sans aucun doute la tâche est facilitée du fait qu'il n'y a pas à se conformer à un type unique de fromage.

Le problème qui se pose en ce qui concerne la coagulation continue est d'obtenir la même structure de gel que dans le bac à fromage, où le lait est au repos et où les micelles de caséine sont toutes reliées entre elles d'une manière identique dans toutes les directions. Un flot uniforme et parfaitement égal peut donner des conditions semblables, mais un récipient de la taille nécessaire pour rendre l'opération possible pendant le temps normal de la coagulation serait alors trop grand et poserait des difficultés sérieuses pour le découpage du caillé. Aussi, les méthodes de coagulation continue doivent tendre à une coagulation rapide.

Une méthode de coagulation continue employée à Ede (Pays-Bas) bénéficie d'une application commerciale limitée en Europe. Elle consiste à séparer les deux phases de l'action de la présure sur le lait, à savoir les modifications d'ordre chimique (scission d'une partie de la caséine) et, en deuxième lieu, la coagulation de la caséine ainsi déstabilisée. Ce procédé hollandais, qui repose sur celui de Berridge en Angleterre, utilise une basse température qui autorise la première modification mais retarde la seconde. La coagulation réelle intervient lors de l'écoulement continu vers le haut après que le lait a été chauffé à sa température de coagulation. Le cylindre de caillé qui apparaît au sommet de la colonne est coupé au fur et à mesure. Les principales difficultés étaient dues à l'aptitude plus ou moins grande du lait à la coagulation, ainsi qu'à une tendance du caillé à s'accumuler sur les côtés des cuves. 
La méthode Hutin-Stenne, qui fait partie du groupe ParacurdAPV, concentre au préalable le lait, l'emprésure à froid, puis le chauffe et le dilue en un même temps, en y ajoutant de l'eau chaude. Le lait se coagule en flocons. Les rapports ioniques à l'intérieur du caillé sont évidemment modifiés dans cette méthode, mais ceci ne saurait être un obstacle à son utilisation pour nous préparer en vue de fabriquer une variété différente de fromages. A cet égard, on doit plutôt se pencher sur la question du prix de revient d'une telle méthode. Il est coûteux de retirer l'eau du lait par évaporation pour ensuite la remettre. Pour certains fromages à pâte molle les frais sont couverts par un rendement meilleur. Ce qu'il faut déterminer - et ceci est lié à "l'autre introduction " à la mécanisation de l'industrie fromagère - c'est de savoir si de semblables augmentations de rendements ne peuvent être obtenues à meilleur marché par d'autres moyens, tels que par exemple un traitement préalable à haute température et peut-être une homogénéisation à basse pression.

Un autre procédé pour séparer les deux étapes de la coagulation du lait, consiste à ôter ou isoler le calcium du lait, à permettre à de la présure de modifier la caséine, puis de réintroduire le calcium. Jusqu'à présent il semble que cette opération qui consiste à isoler le calcium pour ensuite le réintroduire soulève plus de difficultés que l'emploi de basses températures suivies de chauffage rapide. Toutefois on est autorisé à penser que les techniques de coagulation continue peuvent être poussées jusqu'à pouvoir s'intégrer dans un nouveau procédé de fabrication du fromage : du point de vue de l'industrie chimique leur apport peut être des plus fructueux.

\section{Les cultures de levains}

Le projet développé au C.S.I.R.O. en vue d'une production ininterrompue des bactéries de levains vous est maintenant largement connu ; il s'agira d'en approvisionner les usines sous des formes concentrées et congelées pour les ajouter directement dans la cuve. Si ce projet tient ses promesses, comme on est en droit de l'espérer, il est évident que nous devrons penser au procédé nouveau d'addition des levains en vue des nouvelles méthodes de fabrication du fromage. Un système continu en circuit fermé pour l'introduction de ces levains, éventuellement après dilution, pourrait être branché à la sortie de la partie réfrigérante du préchauffeur.

En admettant que ce système soit totalement fermé, comme il doit l'être, et qu'il se prête facilement à un contrôle effectif, le problème des phages dans la fabrication du fromage doit disparaître, grâce à cette nouvelle forme de levain qui en est exempte. Il deviendrait dès lors possible d'utiliser toujours la même souche de germe de levain, qu'il y en ait un ou plusieurs, et l'on pourrait choisir ces germes sur les critères exclusifs de la production d'acide et de la saveur du fromage obtenu. 
Il y a plusieurs années nous avons mis au point une méthode rapide de fabrication du Cheddar (peut-être l'appellation est-elle quelque peu sujette à caution, car à cette époque la nécessité de préserver les caractères distinctifs des différentes variétés de fromages ne nous semblait pas si impérieuse, encore que le produit conservât ses données essentielles). Ce procédé mettait en cuvre, à la place des levains habituels, d'autres streptocoques lactiques qui présentaient la double possibilité de conserver leur action à des températures supérieures à la normale, et de provoquer la fermentation du lactose résiduel du fromage à des taux de concentration saline normaux. Si l'on est débarrassé de la contrainte de fabriquer une sorte de fromage continue, alors il faut examiner en détail toutes les ressources des organismes de levains de ce genre (par exemple Str. Durans). Aux températures plus élevées que l'on peut alors atteindre la plupart des modifications du caillé qui constituent les phases essentielles de la fabrication surviennent plus rapidement.

Revenons à la question de l'amertume. Il est reconnu que ce défaut est largement répandu dans les fromages d'Australie et de Nouvelle-Zélande partout où ils sont vendus, et sur le marché japonais il constitue notre principal souci. C'est avec satisfaction que j'ai aujourd'hui la possibilité de rendre compte d'une explication très récente (elle ne remonte pas à plus de deux semaines) concernant les causes de ce défaut, elle émane du Dr G.R. Jago du C.S.I.R.O. On peut en attendre l'élimination totale de ces effets fâcheux. L'amertume est imputée à des peptides présentant un groupement glutomate terminal. Si l'on arrive à éliminer ce groupe terminal l'amertume disparaîtra. Mais la chaîne résiste aux diastases peptidiques normales. Toutefois nous savons à présent qu'il existe une diastase capable de la scinder, et nous disposons déjà d'une série d'organismes aptes à produire cette diastase en plus ou moins grandes quantités. L'un des procédés possibles pour l'élimination pratique de ce défaut serait de cultiver les organismes appropriés dans une culture continue, de décomposer les cellules de manière à obtenir un extrait, exempt de cellule, ce qui éliminerait des difficultés possibles dues à l'entourage de phage si on utilise les souches tour à tour, et d'ajouter cet extrait dans la cuve à fromage. En ce qui concerne notre nouvelle méthode de fabrication, introduire simplement des quantités assez importantes de ces organismes, là où le levain normal est injecté et par les mêmes méthodes, semble être une première mesure ne présentant pas de difficultés.

Puisque l'on a reconnu la présence en grandes quantités d'autres organismes microcoques et lactobacilles dans le fromage lors de l'affinage et que ceux-ci doivent avoir quelque effet sur la saveur, il est nécessaire non seulement de garantir de bonnes conditions pour leur développement, mais aussi de s'assurer que les souches désirables sont dès le début en majorité ; ainsi au moment où les streptocoques disparaissent pour laisser la place à ces organismes, ces organismes seraient bien ceux que l'on attendait. Il serait donc 
étonnant qu'à la longue on ne trouve pas souhaitable d'ajouter ces organismes au levain. Ce sera d'autant plus nécessaire que le lait aura subi un pré-chauffage plus important.

Comme nous l'avons indiqué à propos des présures microbiennes, il nous est loisible d'anticiper sur notre compréhension des systèmes diastasiques en vue d'une maturation satisfaisante, et il est possible que l'adjonction de telles diastases constitue une part normale de la fabrication fromagère. Toutefois isoler et purifier des diastases représente en soi un travail difficile et coûteux et il semble que l'emploi d'organismes pourvus des diastases adéquates soit plus simple, qu'il s'agisse de les ajouter en tant que cellules vivantes ou en tant que préparations sans cellules.

\section{La synérèse}

L'étape suivante de la fabrication en fromagerie consiste en la réduction de la teneur en humidité des grains de caillé par leur contraction et l'exsudation du sérum, en termes techniques, la synérèse d'une structure de gel. Nous savons déjà d'après les travaux effectués au C.S.I.R.O. qu'un choc entre les grains de caillé accélère considérablement l'opération. C'est la raison pour laquelle l'exsudation de la moitié du sérum est si efficace pour maintenir l'humidité du fromage au niveau voulu, car avec seulement la moitié du sérum, les grains du caillé se heurtent avec une fréquence beaucoup plus grande pendant l'agitation. Cela ressemble à une sorte de secouage de la structure de gel, comme si l'on versait une poudre fine dans un récipient et que l'on frappe sur celui-ci afin que la poudre se tasse. Les faibles contacts qui empêchent les micelles de s'agglomérer sont ainsi brisés par les chocs. Il est possible que ces faibles liens ralentisseurs soient particulièrement sensibles à une énergie vibratoire, ou à la vibration provoquée par les ultra-sons à une fréquence donnée. Des travaux sont entrepris sur cette question. S'ils donnent des réponses affirmatives nous disposerons là d'un moyen facile à contrôler pour activer la synérèse. Si cette méthode est retenue, on doit tenir compte de la nécessité de coordonner la perte en humidité du caillé à, d'une part, la solubilisation du calcium et du phosphore, qui intervient à l'intérieur du caillé sous l'action de l'acide lactique, et, d'autre part, à la transformation du lactose en acide lactique. Si nous prenons un exemple limite, supposons que toute l'eau provenant du grain de caillé ait été recueillie avant la production acide : presque tout le calcium, le phosphore et le lactose demeureraient à l'intérieur du fromage et l'on obtiendrait bien évidemment un fromage acide à pâte courte.

Mais la possibilité de contrôler aisément la teneur en humidité perdue, de même que notre pouvoir, actuellement, de contrôler le taux d'acidification ainsi que l'acidité initiale sans oublier la composition chimique et physique du lait, tout cela permettra à ces différents facteurs d'avoir une interaction pour donner un produit où 
l'on trouverait réunies les conditions nécessaires relatives au corps de la texture, à la saveur initiale et finale. Un autre moyen pour contribuer à la composition idéale consisterait à laver le caillé, comme on le fait pour certaines sortes de fromages, et il n'y a pas de difficulté d'ordre technique à faire de cela une étape dans la fabrication continue ; du reste le procédé est déjà utilisé pour la fabrication de la caséine.

La transformation du lactose en acide lactique à l'intérieur du grain de caillé dure nécessairement un certain temps, et l'on doit prévoir de maintenir le caillé quelque part pendant la durée de l'opération. Maintenir le caillé dans des récipients fixes tout en essayant de le traiter d'une manière continue est mauvais d'un point de vue technique. Il est préférable de faire avancer le caillé sur un transporteur d'égouttage quelconque. Là on a le choix entre, d'une part, le laisser se souder pendant ce temps, auquel cas on doit ensuite le moudre ou le briser de nouveau avant le salage, ou bien, d'autre part, le transformer en fromage et le saumurer, ou bien encore l'empêcher de s'agglomérer en l'agitant, ce qui a l'inconvénient de rendre possible une perte de matière grasse et de fines particules de graisse. L'opération que l'on accomplit pendant ce temps d'arrêt varie suivant que l'on sale le fromage par saupoudrage ou en saumure. Là aussi l'opération est fonction de la méthode retenue pour amener le caillé à la forme définitive du fromage. Considérons donc maintenant les avantages et les inconvénients des différentes solutions possibles en cette phase finale de la fabrication du fromage.

\section{Salage et mise en forme}

Les solutions employées pour réduire la main-d'œuvre considérable que rend nécessaire le pressage du caillé par saupoudrage, telles qu'elles interviennent dans la fabrication de variétés de fromages comme le Cheddar, le Colby, etc., sont les suivantes :

1) Utilisation d'une presse de grandes dimensions où l'on presse d'un seul coup 453 ou $680 \mathrm{~kg}$ (1000 ou 1500 livres) ou même davantage en un seul bloc, puis découpage. On se heurte à des difficultés pour l'élimination de l'humidité ; difficultés aussi concernant l'écoulement sous l'influence de la gravité.

2) Utilisation d'un récipient cylindrique de faibles dimensions en carton-fibre. C'est le procédé que nous avons retenu.

3) Utilisation de la grande presse de Nouvelle-Zélande : procédé qui a suscité de nombreux espoirs, mais où on se heurte à des difficultés qui n'ont pas encore été surmontées.

4) Le procédé en application à Wolvega aux Pays-Bas : On utilise des moules en acier inoxydable d'une capacité de $18,5 \mathrm{~kg}$ (40 livres), munis de repères Perfora en acier inoxydable au lieu de bandes de toile. Ces moules sont automatiquement transportés sur 
des rails, d'abord pendant un laps de temps assez court à une presse travaillant sous vide, où des pistons pneumatiques individuels pressent chaque rangée horizontale de moules, puis de là, à une presse d'un modèle semblable travaillant à la pression atmosphérique et qui achève le pressage en $4 \mathrm{~h}$. Le fromage est sorti par renversement et aussitôt emballé, tandis que le moule est automatiquement lavé et renvoyé pour être réutilisé pendant les mêmes jours de fabrication. A mon avis, c'est jusqu'à présent, la solution la plus attrayante.

Mais à cette même usine des Pays-Bas fonctionne un impressionnant ensemble destiné à saumurer automatiquement des fromages du type Gouda ou Edam. Si le saumurage en soi est beaucoup plus incommode que le salage par saupoudrage, il autorise en revanche une agrégation rapide du caillé dans les moules, ou du moins plus importante dans tout procédé mécanique de mise en forme. Les fromagers connaissent bien les différences qui existent entre le caillé salé par saupoudrage ou à la saumure en ce qui concerne cette agrégation. A Wolvega, les fromages sont automatiquement ôtés des moules en plastique et disposés dans un courant de saumure, puis transportés par la saumure à un compartiment de salage où ils demeurent pendant $6 \mathrm{j}$; ensuite ils sont replacés à nouveau dans le courant de saumure et transportés à une machine qui les soulève, les sèche, les enrobe d'une gaine de matière plastique et les porte à la chambre d'affinage. Ce procédé de saumurage automatique apparaît beaucoup moins encombrant, et aussi plus pratique, dans la mesure où on cesse de faire épouser au caillé une forme donnée après qu'il ait été séché et salé.

Mais une autre manière bien différente d'approcher ce problème serait de placer le fromage dès le début dans des emballages en plastique en portions individuelles. Il semblerait qu'avec cette méthode le salage par saupoudrage soit primordial, mais on peut espérer des progrès de la technologie dans le domaine des feuilles plastiques et qu'il sera possible de rendre la pellicule de plastique capable d'exercer une pression suffisante pour assurer la soudure du caillé après un conditionnement sous vide.

Une autre possibilité en ce domaine serait d'utiliser un enrobage de sel pour le salage par saupoudrage. L'enrobage retarderait la dissolution du sel tant que la soudure du caillé ne serait pas obtenue. Nous avons testé cette méthode, sans grand succès jusqu'à présent.

Ainsi nous sommes en présence d'un certain nombre de solutions dont certaines ont déjà fait l'objet d'applications ; d'autres apparaissent à l'horizon, et nous devons en attendre la mécanisation pour le moulage et l'emballage du fromage. Ces procédés, quels qu'ils soient, introduisant la mécanisation dans la mise en forme du fromage, doivent entrer à part entière dans les nouvelles techniques de fabrication.

(Traduction Jean Thieulin). 